\title{
Sunshine exposure and serum 25OHD concentrations during a 12 month randomized control trial with high dose vitamin $D$ supplementation: results from the Vitamin D Supplementation in Older People (VDOP) study ${ }^{(1)}$
}

\author{
R.M.T.K Ranathunga ${ }^{1,2}$, T.R. Hill ${ }^{1,2}$, J.C. Mathers ${ }^{2,3}$, I. Schoenmakers ${ }^{4}$ and T.A. Aspray ${ }^{3,5}$ \\ on behalf of the VDOP study team \\ ${ }^{1}$ Agriculture Food and Rural Development, Newcastle University, NE1 7RU, \\ ${ }^{2}$ Human Nutrition Research Center, Newcastle University, NE1 7RU, \\ ${ }^{3}$ Institute of Cellular Medicine, Newcastle University, NE4 4PL, \\ ${ }^{4}$ MRC Human Nutrition Research, Cambridge, Medical School, University of East Anglia, Norwich, NR4 7TJ and \\ ${ }^{5}$ Freeman Hospital, Newcastle upon Tyne, NE7 7DN
}

Assessing the relative contribution of sunshine exposure to vitamin D status in randomized control trials with vitamin D supplementation is of paramount importance. The objective of this study was to determine the effect of yearly sun exposure on serum 25OHD concentrations in older adults supplemented with 12 monthly doses of 12000IU, 24000IU and 48000IU vitamin $\mathrm{D}_{3}$.

Serum 25OHD concentration was measured by Tandem Mass Spectrometry at baseline and after 12 month supplementation in 327 adults aged $>70$ years $^{(1)}$. Sunshine exposure of the participants was assessed every three months (baseline, after 3 months, 6 months, 9 months and after 12 months) by a questionnaire which assessed the frequency of outdoor activities, skin exposure area exposed to sun, sunblock usage, holiday visits and Fitzpatrick skin types. Of the 327 participants, $62 \%$ of participants began the 12 months supplementation during the time of year where there is no UVB radiation at the study location $\left(55^{0} \mathrm{~N}\right)$, defined as October to March ${ }^{(2)}$. Composite scores for each sun exposure variable were calculated from the mean of the five questionnaires (including baseline and 12 months follow-up visits). For each treatment arm of the trial, sunshine exposure variables as well as other socio-demographic, lifestyle and dietary factors were included as potential predictors of endpoint serum 25OHD using backward linear regression.

In those participants receiving 12000IU monthly, summer sun exposure, skin exposure area exposed to sun and female gender were positively associated with endpoint vitamin D status while skin type IV, calcium intake and BMI were negatively associated with endpoint vitamin D status. For participants randomized to 24000 IU and 48000 IU monthly, none of the sunshine exposure variables were significantly related to vitamin D status. The proportion of the variance in endpoint serum 25OHD concentration explained by the variables in the model were $36 \%, 21 \%$ and $16 \%$ for the $12000 \mathrm{IU}, 24000 \mathrm{IU}$ and $48000 \mathrm{IU}$ treatment arms, respectively.

\begin{tabular}{|c|c|c|c|c|}
\hline Sun exposure variables & $\beta$ & $\mathrm{SE}$ & $95 \% \mathrm{CI}$ & $\overline{P \text {-value }}$ \\
\hline \multicolumn{5}{|l|}{$12000 \mathrm{IU}$ group $\left(n=100, \mathrm{R}^{2}=0 \cdot 36\right)$} \\
\hline Summer sun exposure (vs winter sun exposure) & $0 \cdot 321$ & $2 \cdot 702$ & $4 \cdot 311,15 \cdot 059$ & $0 \cdot 001$ \\
\hline Skin exposure area & $0 \cdot 396$ & $10 \cdot 017$ & $24 \cdot 234,64 \cdot 080$ & $<0 \cdot 001$ \\
\hline Fitzpatrick skin type IV (vs type I) & $-0 \cdot 207$ & $4 \cdot 134$ & $-17 \cdot 74,-1 \cdot 292$ & $0 \cdot 024$ \\
\hline Calcium intake & $-0 \cdot 185$ & 0.005 & $-0.021,0.000$ & $0 \cdot 041$ \\
\hline Females (vs males) & $0 \cdot 212$ & $2 \cdot 808$ & $0 \cdot 817,11 \cdot 985$ & $0 \cdot 025$ \\
\hline BMI & $-0 \cdot 237$ & $0 \cdot 373$ & $-1.703,-0.219$ & $0 \cdot 012$ \\
\hline \multicolumn{5}{|l|}{$24000 I U$ group $\left(n=112, R^{2}=0 \cdot 21\right)$} \\
\hline Sunblock use & $0 \cdot 191$ & 2.963 & $-0.152,11.633$ & 0.056 \\
\hline BMI & $-0 \cdot 392$ & $0 \cdot 379$ & $-2 \cdot 262,-0 \cdot 754$ & $<0 \cdot 001$ \\
\hline \multicolumn{5}{|l|}{$48000 I U$ group $\left(n=115, R^{2}=0 \cdot 16\right)$} \\
\hline Age & $-0 \cdot 216$ & $0 \cdot 349$ & $-1 \cdot 436,-0 \cdot 050$ & $0 \cdot 036$ \\
\hline BMI & $-0 \cdot 359$ & $0 \cdot 379$ & $-2.048,-0.559$ & $0 \cdot 001$ \\
\hline
\end{tabular}

Endpoint serum 25OHD as dependent variable and frequency of outdoor activities, skin exposure area exposed to sun, Fitzpatrick skin types, sunblock usage, sun exposure season, length of holiday visits, holiday season, calcium and vitamin D intake, age, gender and BMI as dependent variables.

In conclusion, a number of sunshine exposure variables predicted endpoint vitamin D status in participants receiving 12000 IU monthly but vitamin D supplementation appears to overwhelm the effect of sun exposure on 25OHD concentration at higher doses in older adults.

The VDOP study is supported by a grant (MP/ID19544) from Arthritis Research UK and partly supported by Newcastle University and funding from the core programme of the MRC Nutrition and Bone Health Group at MRC Human Nutrition Research funded by the UK MRC, grant code U10596037.

1. Schoenmakers I, Francis M, McColl E et al. (2013) Trials 14, 299

2. Webb A, DeCosta B, \& Holick M (1989) J Clin Endocrinol Metab 68, 882-887. 